\title{
Short-Term Dynamics of a Mixed Chemical and Electrical Synapse in a Rhythmic Network
}

\author{
Akira Mamiya, ${ }^{1}$ Yair Manor, ${ }^{2}$ and Farzan Nadim ${ }^{3}$ \\ ${ }^{1}$ Center for Molecular and Behavioral Neuroscience, Rutgers University, Newark, New Jersey 07102, ${ }^{2}$ Life Sciences Department and Zlotowski Center for \\ Neurosciences, Ben-Gurion University of the Negev, Beer-Sheva, Israel 84105, and ${ }^{3}$ Department of Mathematical Sciences, New Jersey Institute of \\ Technology, and the Department of Biological Sciences, Rutgers University, Newark, New Jersey 07102
}

In the rhythmically active pyloric circuit of the spiny lobster, the synapse between the lateral pyloric (LP) neuron and pyloric constrictor (PY) neuron has an inhibitory depressing chemical and an electrical component. To understand how the dynamics of the LP $\rightarrow$ PY synapse affect the relative firing times between these two neurons in an ongoing rhythm, we characterized the dynamics of the LP $\rightarrow \mathrm{PY}$ synapse after a pharmacological block of ongoing activity. When a train of voltage pulses was applied to the voltage-clamped LP neuron, the inhibitory chemical component of the postsynaptic potential (PSP) in the PY neuron rapidly depressed. Thus, after the first few pulses, the PSP was either hyperpolarizing or depolarizing, depending on the interpulse duration, with shorter interpulse durations producing depolarizing PSPs. To characterize the synaptic response during rhythmic activity, we played back prerecorded realistic waveforms in the voltage-clamped LP neuron. After an initial transient, the resulting PSP in PY was always depolarizing, suggesting that in an ongoing rhythm, the electrical component of the synapse is dominant. However, our results indicate that the chemical component of the synapse acts to delay the peak time of the PSP and to reduce its amplitude, and that these effects become more important at slower cycle periods.

Key words: synaptic depression; synaptic dynamics; stomatogastric ganglion; oscillations; central pattern generator; motor system

\section{Introduction}

Neurons coupled with electrical synapses often form chemical synapses as well (Michelson and Wong, 1994; Benardo, 1997; Gibson et al., 1999; Mann-Metzer and Yarom, 1999; Fukuda and Kosaka, 2000). The composite "mixed synapse" is considered a single functional unit. Several studies have proposed that mixed synapses act to synchronize the firing of neurons (Benardo, 1997; Galarreta and Hestrin, 1999; Tamas et al., 2000) or serve as a coincidence-detection mechanism of excitatory inputs (Galarreta and Hestrin, 2001a). Mixed synapses have also been implicated in neuronal rhythmogenesis (Galarreta and Hestrin, 2001b). In many mixed synapses, the chemical component shows short-term facilitation or depression, whereas the effect of electrical synapses is less use dependent (Galarreta and Hestrin, 1999; Gibson et al., 1999; Tamas et al., 2000). Thus, a mixed synapse might dynamically change its properties in response to a modification in the frequency or pattern of activation of the synapse (Galarreta and Hestrin, 2001b).

We explore this possibility using the rhythmically active pyloric network (frequency, 0.5-2 Hz) of the spiny lobster Panulirus interruptus. The pyloric rhythm is generated by a pacemaker ensemble of an anterior burster $(\mathrm{AB})$ and two pyloric dilator (PD) neurons. The remaining (follower) neurons are coupled to the

\footnotetext{
Received June 24, 2003; revised Aug. 15, 2003; accepted Aug. 28, 2003.

This research was supported by United States-Israel Binational Science Foundation Grant 2001-039 (Y.M. and F.N.) and National Institute of Mental Health Grant 60605-01 (F.N.).

Correspondence should be addressed to Farzan Nadim, Department of Biological Sciences, 101 Warren Street, Newark, NJ 07102. E-mail: farzan@njit.edu.

Copyright $\odot 2003$ Society for Neuroscience $\quad$ 0270-6474/03/239557-08\$15.00/0
}

pacemaker neurons with electrical and inhibitory chemical synapses. The period and activity phase of pyloric neurons are determined by the intrinsic properties of these neurons as well as the dynamics of the synapses among them (Nusbaum and Beenhakker, 2002).

An important property of the pyloric network is that the relative phase of activity of different neurons in the network is adjusted depending on the cycle frequency to maintain a stable triphasic pattern (Miller and Selverston, 1982). Two classes of follower neurons, the lateral pyloric (LP) and pyloric constrictor (PY) neurons, are reciprocally coupled via mixed synapses. In the transmission of signals from the LP neuron to the PY neuron, the two components of the mixed synapse have opposite effects. When the LP neuron bursts, the electrical component promotes PY neuron activity, whereas the chemical component acts to suppress it. Moreover, previous studies have shown that the relative phase of activity of the LP and PY neurons was well maintained when the frequency of the pyloric rhythm was altered by external manipulation (Hooper, 1997a,b).

In this study, we explore the complex interaction between electrical and inhibitory chemical components in the $\mathrm{LP} \rightarrow \mathrm{PY}$ synapse. We first characterize parameters describing the dynamics of the synapse (amplitude and the extent and time constant of depression) by suppressing the pyloric activity, voltage clamping the LP neuron with trains of voltage pulses, and recording the postsynaptic potential (PSP) in the PY neuron. After determining the synaptic parameters, we describe the synaptic activity in the context of the rhythmic pyloric rhythm. This is done by playing back prerecorded realistic waveforms in the voltage-clamped LP neuron and recording the PSP. Finally, using correlation analysis, 
we show that the frequency-dependent properties of the LP $\rightarrow$ PY mixed synapse are important in determining the firing phase of the PY neurons during rhythmic activity. These results indicate that dynamic properties of a mixed synapse can serve as a mechanism for fine-tuning of phasing in oscillatory networks.

\section{Materials and Methods}

All experiments were done on adult spiny lobsters P. interruptus (Don Tomlinson Fisheries, San Diego, CA).The stomatogastric nervous system (STNS) was isolated with standard procedures (Selverston et al., 1976; Harris-Warrick et al., 1992). The isolated STNS was pinned down on a Sylgard-coated Petri dish and superfused with chilled $\left(16^{\circ} \mathrm{C}\right)$ physiological saline containing the following (in $\mathrm{mm}$ ): $479 \mathrm{NaCl}$, $12.9 \mathrm{KCl}, 13.7 \mathrm{CaCl}_{2} \cdot 2 \mathrm{H}_{2} \mathrm{O}, 10 \mathrm{MgSO}_{4} \cdot 7 \mathrm{H}_{2} \mathrm{O}$, $3.9 \mathrm{NaSO}_{4} \cdot 10 \mathrm{H}_{2} \mathrm{O}, 11.2$ Trizma base, and 5.1 maleic acid, pH 7.45.

Pyloric neurons were identified by their stereotypical axonal projections in identified nerves using conventional techniques (Selverston et al., 1976; Harris-Warrick et al., 1992). The pyloric activity was monitored with stainless-steel wire electrodes from identified nerves. Extracellular signals were amplified with a differential AC amplifier (model 1700; A-M systems, Carlsborg, WA). Intracellular recordings were made by impaling the soma with sharp microelectrodes filled with $0.6 \mathrm{M} \mathrm{K}_{2} \mathrm{SO}_{4}$ plus $20 \mathrm{~mm} \mathrm{KCl} \mathrm{(25-35}$ $\mathrm{M} \Omega$ for identification of neurons and for recording the postsynaptic neuron) or $3 \mathrm{M} \mathrm{KCl} \mathrm{(8-12} \mathrm{M} \Omega$ for current injection in the presynaptic neuron). All intracellular recordings were done with Axoclamp 2B amplifiers (Axon Instruments, Foster City, CA).

Synaptic potentials were measured after abolishing the pyloric activity. This was done by bath application of $0.1 \mu \mathrm{M}$ tetrodotoxin (TTX) (Biotium, Hayward, CA) to block both descending inputs to and activity within the stomatogastric ganglion. As expected, TTX eliminated the spike-mediated component of the $\mathrm{LP} \rightarrow \mathrm{PY}$ synapse. For technical reasons, we measured synaptic transmission in TTX so that presynaptic release could be precisely controlled. In the pyloric network, an important component of synaptic transmission is graded (Graubard et al., 1980), and oscillatory activity could be produced in the absence of action potentials (Raper, 1979). Synaptic activity was studied by voltage clamping the presynaptic neuron using a series of voltage pulses or realistic waveforms from a holding potential of $-60 \mathrm{mV}$. The postsynaptic response was monitored in current-clamp mode. The $\mathrm{LP} \rightarrow \mathrm{PY}$ synapse has both electrical and chemical components. Because there is no known specific blocker for the electrical coupling in this system, the chemical component was estimated by subtracting the postsynaptic potential recorded after blocking the chemical synapse from that recorded under control conditions. Such subtraction of voltages presupposes a linear $I-V$ relationship. Because the amplitude of the recorded PSPs was relatively small, this subtraction provides a good estimate of the chemical component. The chemical synapse was blocked by bath application of $10 \mu \mathrm{M}$ picrotoxin (PTX) (Marder and Paupardin-Tritsch, 1978).

Realistic waveforms were constructed by recording voltage traces of the LP neuron in an ongoing rhythm. To obtain typical LP neuron waveforms at different pyloric periods, we injected current into one of the pacemaker group neurons (AB-PD) to change the period. We chose two representative cases, recorded from a single experiment, with short (625 $\mathrm{msec})$ and long $(1600 \mathrm{msec})$ periods. For each case, we low-pass filtered the trace at $10 \mathrm{~Hz}$, divided the trace to single cycles, and averaged these cycles to construct a unitary waveform. In a quiescent LP neuron, we repeatedly injected the unitary waveform to stimulate the LP neuron with a realistic waveform. The same LP neuron waveforms were used in all experiments. A comparison with LP neuron waveforms recorded from several experiments shows that these waveforms are representative of the LP neuron activity (data not shown). Note that the fact that we filter the waveforms and the filtering caused by the electrotonic structure of the LP neuron cause the chemical component of the synapse to be underestimated. This point is addressed in the Discussion. Manipulations of traces (waveform cutting, low-pass filtering, and averaging) were done with a custom-made program written in LabWindows/CVI (National Instruments, Houston, TX).

All intracellular recordings were digitized at $4 \mathrm{kHz}$ and stored on a personal computer using a PCI-MIO-16E-1 board (National Instruments) and custom-made recording software written in LabWindows/ CVI (National Instruments). All analyses, such as detection of the peak time and the amplitude of the postsynaptic response, averaging the synaptic response, calculation of the ratios, digital subtraction of the traces, and curve fitting, were done by custom-made programs written in Matlab (Mathworks, Natick, MA). Statistical tests were done using SAS package (SAS Institute, Cary, NC).

\section{Results}

Neurons that participate in the pyloric rhythm fire bursts of action potentials in three distinct phases. The pacemaker $A B$ and PD neurons fire in-phase, followed by, after a short delay, a burst of action potentials in the LP neuron and then followed by, with some overlap, bursts of action potentials in the PY neurons (Fig. $1 A)$. The period and firing phases of neurons in the pyloric network are determined by the intrinsic properties of these neurons as well as the dynamics of the synapses among them (Nusbaum and Beenhakker, 2002). Figure $1 B$ shows the connectivity among the $\mathrm{AB}, \mathrm{PD}, \mathrm{LP}$, and PY neurons. In this study, we explore the interaction between electrical and inhibitory chemical components of the mixed synapse between the LP and PY neurons. Previous studies have shown that the electrical component of this synapse is rectifying: it allows preferential flow of positive current from the LP neuron to the PY neuron (Graubard and Hartline, 1987; Johnson et al., 1993). We show that the inhibitory chemical component of this synapse is strongly depressing and examine 

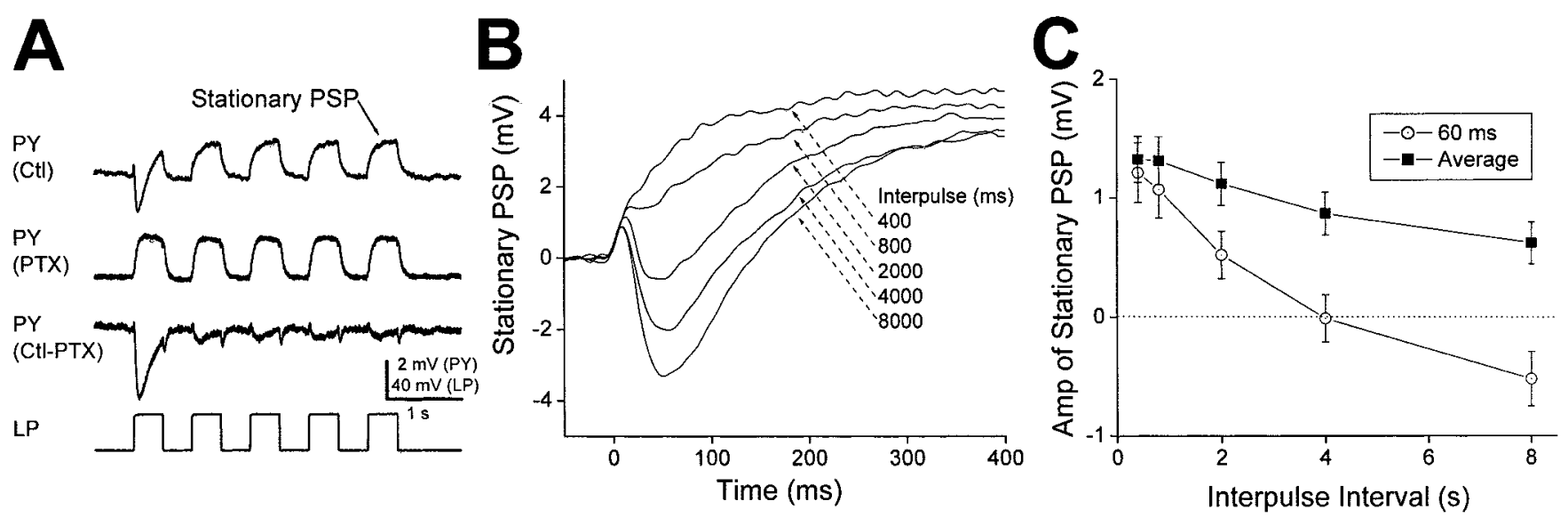

Figure 2. Postsynaptic potential of PY in response to stimulation of the $L P$ neuron with trains of voltage pulses. $A, A$ train of five $40 \mathrm{mV}$ voltage pulses were injected in the $L P$ neuron. Control (CtI) and PTX traces show the voltage response of a PY neuron in controls and in $10 \mu \mathrm{m}$ picrotoxin. The Ctl - PTX trace shows the chemical component of the LP $\rightarrow$ PY synapse obtained by subtracting the $\mathrm{Ct}$ and PTX traces. B, The voltage response of the PY neuron at the fifth pulse was defined as the stationary PSP. Shown are stationary PSPs for different interpulse durations (400 and $800 \mathrm{msec}$ and 2,4 , and $8 \mathrm{sec}$ ). C, Amplitude of the stationary PSP (mean \pm SEM; $n=18$ ) as a function of the interpulse interval measured at 60 msec from the pulse onset (open circles) and as average (integral-pulse duration, filled squares). Dotted line denotes $0 \mathrm{mV}$.

the dynamics resulting from the combination of these two components.

Figure $1 C$ shows how the burst onset phase was calculated for each PY neuron. The burst onset phase was defined as the time difference $(\Delta \mathrm{T})$ between the LP neuron burst onset and the PY neuron burst onset, divided by the ongoing pyloric cycle period $\left(\mathrm{P}_{\text {natural }}\right)$. Finally, we examined dynamics and function of the $\mathrm{LP} \rightarrow \mathrm{PY}$ synapse in a setting closer to that of an ongoing rhythm by voltage clamping the LP neuron using prerecorded realistic waveforms and comparing the timing of the PSP with the burst timing of the postsynaptic PY neuron.

\section{Characterization of the dynamics of the $\mathrm{LP} \rightarrow \mathrm{PY}$ synapse with trains of voltage pulses}

To study the dynamic properties of the $\mathrm{LP} \rightarrow \mathrm{PY}$ synapse and to determine the parameters for these dynamics (for example, magnitude of depression and time course of recovery), we first activated the synapse with trains of voltage pulses and examined the dynamics of PSP.

Figure $2 A$ is an example showing voltage traces of the LP and PY neurons under this paradigm. In this example, both the pulse and the interpulse durations were $400 \mathrm{msec}$. The top trace shows the response of the PY neuron under control conditions. The second trace is the PY response in the presence of PTX and represents the electrical component of the synapse. The chemical component of the synapse was approximated by subtracting the second trace from the first trace (Fig. $2 \mathrm{~A}$, third trace, Ctl-PTX).

In control saline, the PY response to the first pulse consisted of three phases: a brief and small depolarization, a $2 \mathrm{mV}$ hyperpolarization that peaked at $60 \mathrm{msec}$, and a depolarization that was truncated at the end of the pulse. In subsequent pulses, the membrane potential of the PY neuron was purely depolarizing and reached steady state near the end of the pulse. The depolarizing and hyperpolarizing phases of the PY response were attributable to the electrical and chemical components of the LP and PY synapse, respectively. In PTX, all five pulses in the LP neuron produced depolarizing responses in the PY neuron, each of which reached steady-state level after $<100 \mathrm{msec}$. The electrical coupling coefficient for the $\mathrm{LP} \rightarrow \mathrm{PY}$ synapses tested $\left(\Delta \mathrm{V}_{\mathrm{PY}} / \Delta \mathrm{V}_{\mathrm{LP}}\right.$ in PTX) was $0.0469 \pm 0.0065$ (mean \pm SEM; $n=18$ ).

The chemical component of the synapse approximated by subtraction (Ctl-PTX) consisted of a $3 \mathrm{mV}$ hyperpolarization in response to the first pulse, followed by smaller $(<0.5 \mathrm{mV})$ hyperpolarizations in response to subsequent pulses. The amplitude difference between the first and subsequent responses indicated that the chemical component showed strong depression. Note that the subtraction of the PTX trace from the control trace produced small transients at the beginning and end of each pulse. These transients were a result of the fact that the PY response was faster in PTX than in control.

To examine the dynamics of the LP $\rightarrow$ PY synapse, we measured the dependence of the PY neuron PSPs on the durations of the interpulse intervals (IPIs). In general, the responses to the fourth and fifth pulses were identical, indicating that the PSP had reached a stationary state. We thus defined the response to the fifth pulse as the stationary PSP. Figure $2 B$ shows the stationary $\mathrm{PSP}$ in response to activation of the $\mathrm{LP} \rightarrow \mathrm{PY}$ synapse with a train of voltage pulses for five different interpulse intervals. As we increased the interpulse interval and allowed more time for recovery from depression, the stationary PSP became less depolarizing. In Figure $2 B$, the PSP was purely depolarizing when the interpulse interval was $\leq 800 \mathrm{msec}$. However, with longer interpulse intervals, the PSP had a hyperpolarizing component. As a result, with longer interpulse intervals, the depolarizing PSP at the end of the pulse was also smaller.

Figure $2 B$ shows that the negative peak of the PY neuron PSP disappeared as the interpulse interval was decreased, thus indicating that the synapse could effectively change sign from negative to positive when the frequency of the presynaptic signal was increased. The negative component of the PSP typically peaked at $\sim 50-70 \mathrm{msec}$ after the beginning of the pulse. We thus measured the amplitude of the PSP at $60 \mathrm{msec}$ as a function of the interpulse duration (Fig. $2 C$, open circles). This amplitude showed a significant change with interpulse duration (one-way ANOVA; $n=18$; $p<0.0001)$ and changed from positive to negative at an interpulse duration of $\sim 4 \mathrm{sec}$. In several cases, the PSP at $60 \mathrm{msec}$ was positive ( 5 of 18 cases) or negative ( 2 of 18 cases) in sign at all measured interpulse intervals (data not shown).

The amplitude of the PSP at $60 \mathrm{msec}$ gave us a measure of the strength of the PSP at the beginning of the pulse only. As a more general measure of the strength of the PSP, we calculated the average amplitude of the stationary PSP by integrating the PSP 


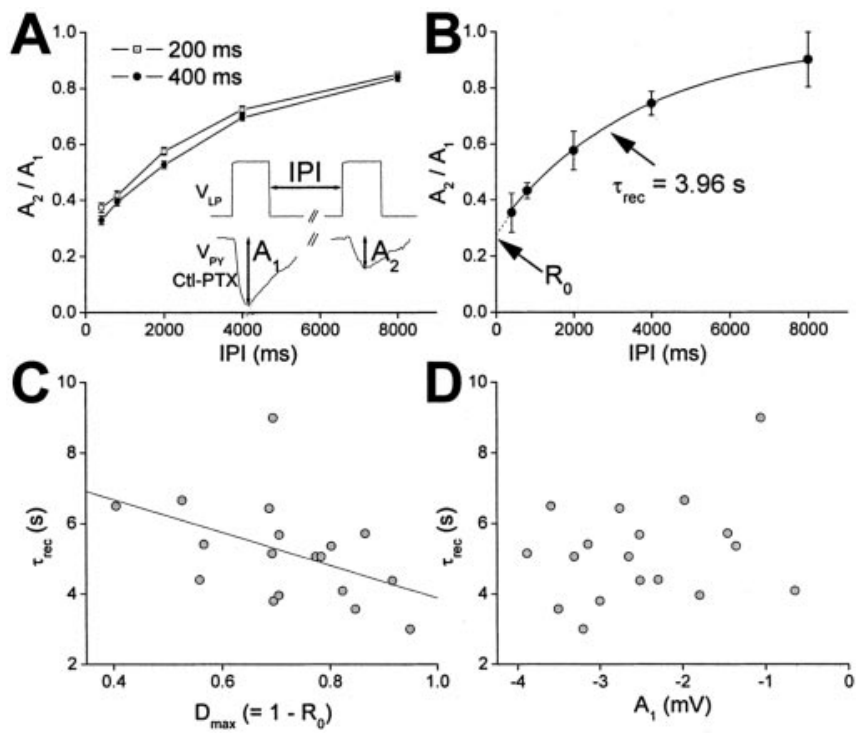

Figure 3. Recovery and depression of the chemical component of the $L P \rightarrow P Y$ synapse. $A, A$ paired-pulse paradigm was used to measure the extent of synaptic recovery as function of interpulse interval (inset). All presynaptic pulses were $40 \mathrm{mV}$ in amplitude. The peak ratio of the second chemical IPSP $\left(A_{2}\right)$ to the first chemical IPSP $\left(A_{1}\right)$ was plotted as function of IPI for two different pulse durations (mean $\pm \mathrm{SEM} ; n=18$; open squares, $200 \mathrm{msec}$; filled circles, 400 msec). (tl, Control. $B$, In each experiment, the ratio $A_{2} / A_{1}$ versus IPI was fit with a single exponential fit. $C$, Time constant of recovery as function of maximum level of depression (mean $\pm \mathrm{SEM} ; n=18$ ). The solid line represents a linear correlation. $D$, The time constant of recovery did not show any correlation with the peak chemical IPSP.

waveform during the presynaptic pulse and dividing by the pulse duration. Figure $2 C$ (filled squares) shows these data for a pulse duration of $400 \mathrm{msec}$. This plot indicated that the average PSP also decreased with interpulse duration (one-way ANOVA; $n=$ 18; $p<0.0001$ ) but remained positive at all durations measured.

Figure 2 shows that the $\mathrm{LP} \rightarrow \mathrm{PY}$ synapse changed sign as a function of interpulse interval. These dynamics arose from an interaction between an electrical and a chemical component. Using the pulse train paradigm described above, we found that the amplitude of the PY responses in PTX did not change even at the shortest interpulse interval (one-way ANOVA; $n=18 ; p>0.15$ ) (Fig. $2 \mathrm{~A}$, second trace). This suggested that the electrical component does not show synaptic depression or facilitation at the interpulse intervals that we tested. Hence, we focused on characterizing the frequency dependence of the chemical component.

Activating the synapse with the same voltage step injected at different interpulse intervals allowed us to quantify the dynamics of the chemical IPSP (control - PTX) using paired-pulse paradigm. Figure $3 A$ shows the ratio of the second peak $\left(A_{2}\right)$ to the first peak $\left(A_{1}\right)$ of the chemical IPSP (see inset) as a function of the IPI for pulse durations of 200 and $400 \mathrm{msec}$ (mean \pm SEM; $n=$ 18). At any IPI, the extent of recovery from depression was larger for the shorter $(200 \mathrm{msec})$ pulses (two-way ANOVA; $p<0.0001$ ), indicating that there was more recovery when the synapse was activated for a shorter duration. However, this difference was small and probably not functionally significant under control conditions.

The relationship between recovery and the IPI is a measure of the time constant of recovery from depression. To quantify this time constant, in each experiment, we computed the relationship of recovery and the IPI and fit it with an exponential curve with time constant $\tau_{\text {rec }}$. In the example shown in Figure $3 B$ (mean $\pm \sigma$ across five trials), $\tau_{\text {rec }}$ was $3.96 \mathrm{sec}$. The $y$-intercept of the expo- nential fit $\left(R_{0}\right.$, as indicated by the arrow) shows the extent of recovery as IPI tends to zero. Thus, $D_{\max }=1-R_{0}$ is a measure of the maximum level of depression for trains of pulses of given duration and amplitude ( $400 \mathrm{msec}$ and $40 \mathrm{mV}$, respectively) (Fig. $3 B$ ). The exponential fit is given by the following equation:

$$
1-D_{\max } e^{-\mathrm{IPI} / \tau_{\mathrm{rec}}}
$$

Note that $D_{\max }=0$ corresponds to a nondepressing synapse. Figure $3 C$ is a scatter plot of $\tau_{\text {rec }}$ versus $D_{\max }$ for 18 PY neurons. As seen in this figure, both parameters were highly variable for different PY neurons. There was a weak negative correlation between the two parameters $(y=-4.7 x+8.5 ; r=-0.469 ; p<$ $0.05 ; n=18$ ), indicating that the synapses that depressed more recovered faster. Surprisingly, there was no correlation between the peak amplitude of the initial IPSP (Fig. $3 A$, inset, $A_{1}$ ) and $\tau_{\text {rec }}$, indicating that stronger synapses did not recover faster or more slowly (Fig. 3D). There was also no correlation between $A_{1}$ and $D_{\max }$ (data not shown).

Although it has been shown that PY neurons can be divided into two groups according to their oscillation phase (Hartline and Gassie, 1979; Eisen and Marder, 1984), we did not observe any clear division of the time constant and maximum depression of the measured PSPs into two groups. Rather, these parameters were continuously distributed.

\section{Correlations between the characteristics of the LP $\rightarrow$ PY PSP and the PY burst phase}

To investigate the effect of the $\mathrm{LP} \rightarrow \mathrm{PY}$ synapse on the activity of the PY neuron in realistic situations, we measured the correlation of the PY burst phase during the ongoing pyloric rhythm (Fig. $1 C$ ) to several characteristics of the PY neuron PSP (measured after the rhythm was abolished with TTX). We measured the PY burst phase in each preparation during the ongoing pyloric rhythm, before the application of TTX.

Figure $4 A, B$ are scatter plots of the PY burst phase $\left(\Delta \mathrm{T} / \mathrm{P}_{\text {natural }}\right)$ versus peak chemical IPSP and maximum electrical EPSP, respectively (see arrows in inset). The PY burst phase did not show any significant correlation with either parameter, nor was there any correlation between the peak time of the chemical IPSP and the PY phase (data not shown). In contrast, Figure $4 C$ shows that the ratio of the peak chemical IPSP to the maximum electrical EPSP was negatively correlated with the PY burst phase $(y=-0.15 x-0.04$; $r=-0.72 ; p<0.01)$.

The ratio of the peak chemical IPSP to the maximum electrical EPSP is a measure of the relative strengths of the chemical and electrical components of the PSP. This measure, however, does not take into consideration the relative time courses of these two components. To account for the kinetics of the two components, we measured the time at which the first PSP reversed from hyperpolarizing to depolarizing, because all PY synapses tested changed sign during the first pulse. We refer to this time as the zero-cross time (see inset). Figure $4 D$ shows that the PY neuron phase is strongly correlated with the zero-cross time of the PSP $(y=-0.0009 x+0.02 ; r=0.854 ; p<0.001)$.

The correlations between the PY phase and both the zerocross time and the ratio of the peak chemical IPSP to the maximum electrical EPSP indicated that the relative strength and kinetics of the two PSP components could reliably predict the contribution of the LP neuron to the bursting phase of the PY neuron. Thus, those PY neurons with a PSP that switched sign at a later time or had a strong chemical versus electrical component tended to burst at a later phase. 

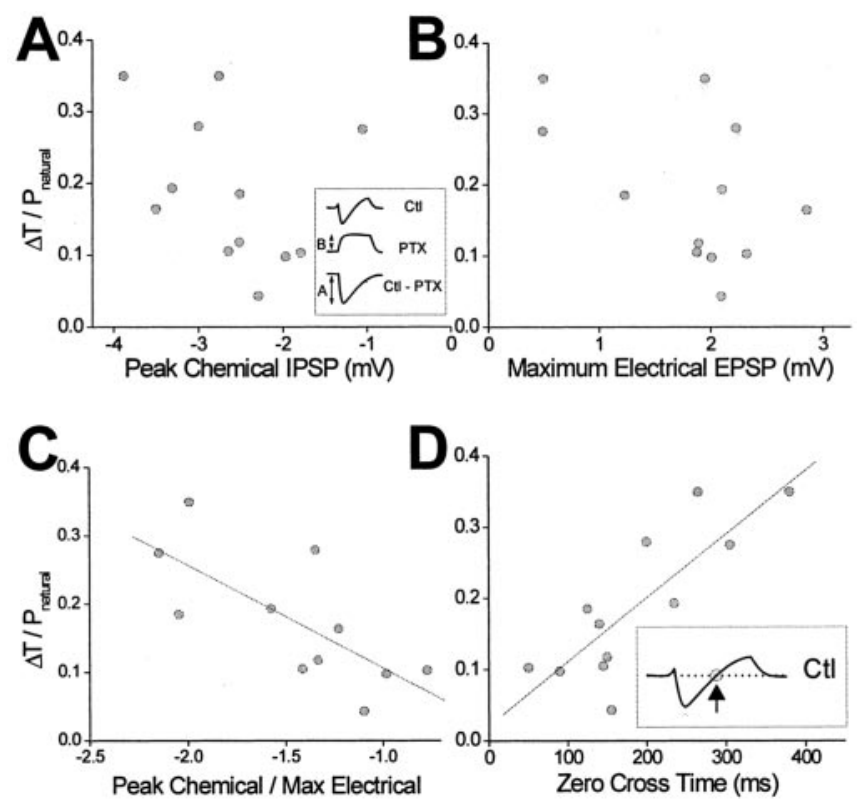

Figure 4. PY burst phase correlates with some dynamic parameters of the $L P \rightarrow P Y$ synapse. PY burst phase was measured in the ongoing pyloric rhythm, as the ratio of $\Delta T$ and $P_{\text {natural }}$ (the pyloric period). Schematic insets show how these parameters were obtained from PY PSP responses. The PY burst phase was not correlated with the peak chemical IPSP $(A)$ or the maximal electrical EPSP $(B)$ but was strongly correlated with the ratio of the peak chemical IPSP to the maximal electrical EPSP $(C)$ and the zero cross time $(D)$. Ctl, Control. Solid diagonal lines are best linear fits.

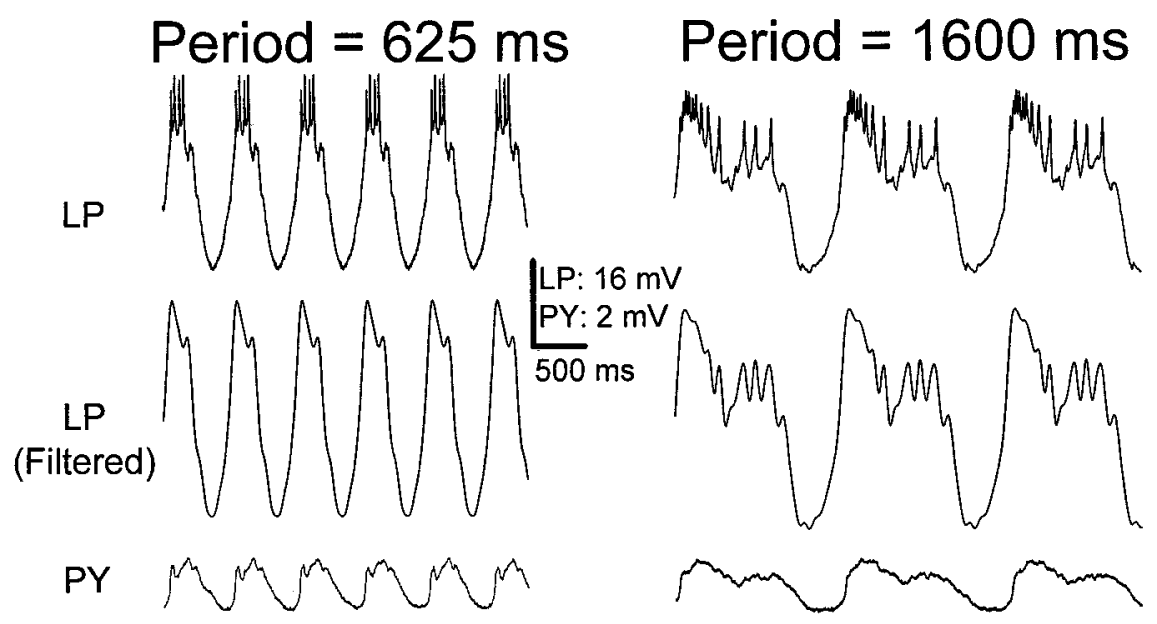

Figure 5. Construction of realistic waveforms. The pyloric frequency was modified by injecting current into the pacemaker neurons (AB-PD). Typical short-period ( $625 \mathrm{msec}$, top left trace) and long-period (1600 msec, top right trace) waveforms were recorded in the LP neuron and low-pass filtered at $10 \mathrm{~Hz}$ to produce smooth unitary waveforms (middle traces). The resulting waveforms were played back into the voltage-clamped LP neuron (see Materials and Methods), and the postsynaptic potentials were measured in the PY neuron (bottom traces).

Traditionally, the firing phase of individual pyloric neurons is computed with respect to the burst onset of the pacemaker neurons $\mathrm{AB}$ and $\mathrm{PD}$. However, in our study, there was no significant correlation between the parameters of the LP $\rightarrow$ PY PSP and the PY burst phase in an ongoing rhythm when measured with respect to the PD neuron (data not shown). This is not surprising, because the timing between the burst of the PD neuron and the PY neuron can be influenced by many factors other than the $\mathrm{LP} \rightarrow \mathrm{PY}$ synapse, such as the synapse from the AB neuron and the PD neuron to the PY neuron (Eisen and Marder, 1984).
Synaptic dynamics in response to the realistic waveforms Until now, we investigated the properties of the LP $\rightarrow$ PY synapse by activating the synapse with square voltage pulses. However, graded synapses are known to show different responses depending on the shape of the membrane potential waveform of the presynaptic cell (Olsen and Calabrese, 1996; Manor et al., 1997; Simmons, 2002). Hence, to examine the role of the LP $\rightarrow$ PY synapse in realistic conditions, we studied the dynamics of the synapse by activating it with realistic LP neuron waveforms. Two sets of realistic waveforms were constructed (see Materials and Methods) by recording LP neuron voltage traces during slow and fast pyloric rhythms and low-pass filtering the waveform at $10 \mathrm{~Hz}$ (Fig. 5, top two traces). Only these two waveforms (with the cycle period indicated) were used in the current study.

After the ongoing rhythm was abolished with bath application of TTX, 10 repetitions of the resulting waveforms were played back into the LP neuron in voltage clamp (from a holding potential of $-60 \mathrm{mV}$ ) and the PSPs were recorded from the PY neuron. Each waveform was always applied at the same cycle period (indicated in Fig. 5) that it was recorded. We will refer to this period as $\mathrm{P}_{\text {applied }}$. Figure 5 (bottom traces) shows an example of the PSPs recorded from the PY neuron when the $\mathrm{LP} \rightarrow \mathrm{PY}$ synapse was activated with repetitive applications of realistic LP neuron waveforms. The initial small peak in the PSPs in response to the shortperiod waveform was seen in a few cases and was caused by the slight delay of the activation of the chemical synapse with respect to the electrical synapse.

The PSP in response to the first one or two cycles of the waveform was different in shape (compared with subsequent cycles) and occasionally hyperpolarizing (data not shown). However, after a few cycles of the waveform, the individual PSPs in response to each cycle of the realistic waveform were identical in shape and size and always depolarizing. In our analysis, we use the PY PSPs measured after the fifth cycle of the realistic waveform.

We examined the role of chemical inhibition by comparing the PSP recorded in the absence of chemical synapse (marked PTX) with those recorded under control conditions. Figure $6 \mathrm{~A}$ shows an example of such a comparison for the short-period LP neuron waveform $\left(\mathrm{P}_{\text {applied }}=625 \mathrm{msec}\right)$. Although in both control and PTX, the PSPs were depolarizing, their peak times (measured from the peak of the LP neuron waveform to the peak of the PSP) and peak amplitudes were different. In PTX, the peak PSP occurred earlier and was larger, suggesting that the chemical component of the synapse acted to delay and decrease the amplitude of the PSP ( $p<0.005$; paired $t$ test; $n=9$ for both the delay of the peak and the reduction of the amplitude) (Fig. 6B). Similar results were obtained with the long-period $\left(\mathrm{P}_{\text {applied }}=1600 \mathrm{msec}\right)$ LP neuron waveform (Fig. 6B). When the PSP peak times were normalized to the respective $\mathrm{P}_{\text {applied }}$, the resulting PSP peak phases showed that the PSPs under control conditions were closer than those in PTX (Fig. 6C). This indicated that the chemical component allowed the synapse peak at a similar phase, independent of $\mathrm{P}_{\text {applied }}$.

One of our main hypotheses is that the dynamics of the $\mathrm{LP} \rightarrow \mathrm{PY}$ synapse, measured in the absence of pyloric activity, are 

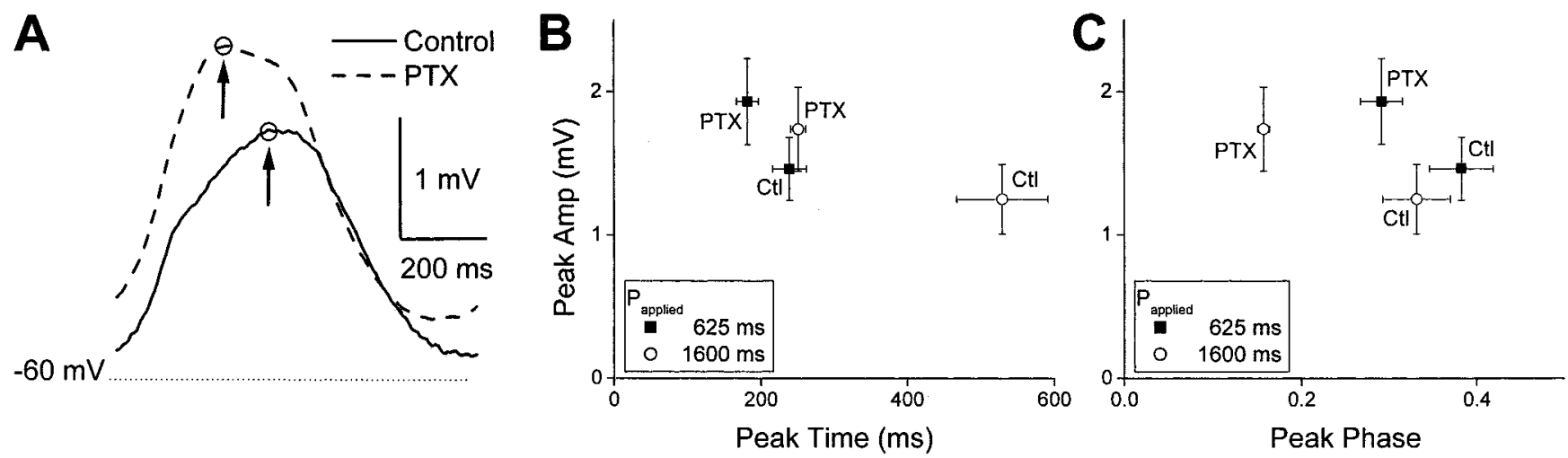

Figure 6. The LP $\rightarrow P Y$ chemical inhibition decreases the amplitude and delays the peak of the PSP in PY. A, A prerecorded realistic LP neuron waveform was played back in the voltage-clamped LP neuron and the PY PSP was recorded under control conditions (solid line) and in the presence of $10 \mu \mathrm{m}$ picrotoxin (dashed line). The example shown is the response to a short-period waveform $\left(P_{\text {applied }}, 625 \mathrm{msec}\right)$. Circles and arrows label the PSP peak amplitudes and peak times. Horizontal dotted line is $-60 \mathrm{mV}$. B, The peak amplitude (Amp) and peak time were significantly different in control and in PTX $(p<0.005 ; t$ test; $n=9)$ at both periods. C, The data in $B$ normalized to the applied period show that the peak PSPs were closer in phase in control compared with PTX. Ctl, Control.
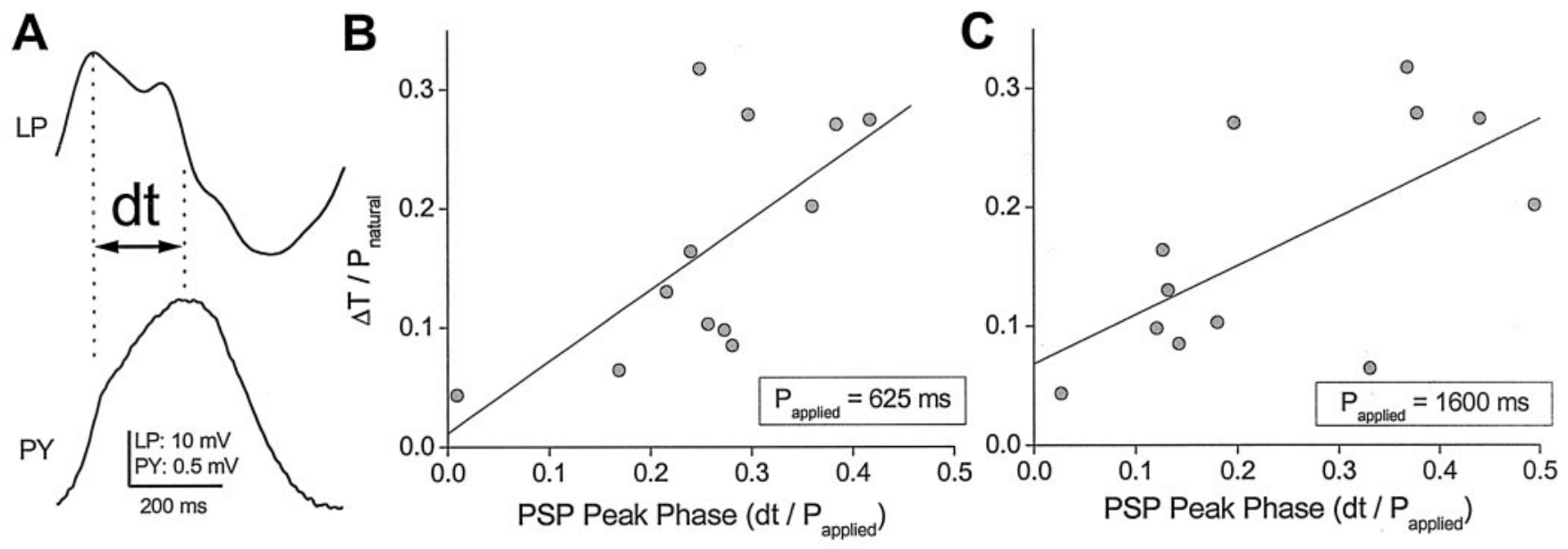

Figure 7. Correlation between the phase of the PY PSP and the phase of the PY burst in an ongoing rhythm. $A$, The phase of the PY PSP was measured as the ratio of the interval between the peak times of the LP neuron waveform and the PY PSP ( $d t)$ to the cycle period $\left(P_{\text {applied }}\right)$. Vertical dotted lines denote the peak of the LP waveform and the PY PSP. B, The PY burst phase in the ongoing rhythm (see Results and legend to Fig. 4) was plotted against the PY PSP phase when the LP neuron was injected with a short-period waveform ( $\left.P_{\text {applied }}, 625 \mathrm{msec}\right)$. Also shown is the linear regression fit. C, Same as B, when the LP neuron was injected with a long-period waveform ( $\left.P_{\text {applied }}, 1600 \mathrm{msec}\right)$. Solid diagonal lines in $B$ and $($ are best linear fits.

important in determining the phasing of the PY neurons during the natural ongoing pyloric rhythm. A direct examination of this contribution was not possible, because we recorded the PSP in PY neurons after blocking rhythmic pyloric activity and playing back the LP neuron waveform in the voltage-clamped LP neuron. However, when we compared the peak phase of the PY PSP (when measured in the absence of activity) with the PY phase in an ongoing natural pyloric rhythm, we obtained a net correlation, which supported our hypothesis.

This correlation analysis is shown in Figure 7. Each data point in $B$ and $C$ corresponds to two phase measurements calculated in the same preparation. The first measurement was taken at the beginning of an experiment during a natural, ongoing pyloric rhythm. At this time, the cycle period and the time difference between the onset of burst activity in the LP neuron and the PY neuron were measured. Using these measurements, the PY neuron phase $\left(\Delta \mathrm{T} / \mathrm{P}_{\text {natural }}\right)$ in the ongoing pyloric rhythm was calculated. The second measurement was taken after bath application of TTX, which abolished the pyloric rhythm. At this time, the LP neuron was voltage clamped with a realistic waveform, applied with fixed cycle period $\left(\mathrm{P}_{\text {applied }}\right)$. The postsynaptic potential was recorded in the PY neuron, and the interval $(\mathrm{dt})$ between the peak of the PY PSP and the peak of the injected realistic LP neuron waveform was measured (Fig. 7A). Using these measurements, the phase of the PY PSP, defined as $\Delta \mathrm{T} / \mathrm{P}_{\text {applied }}$, was calculated.

Figure $7 B, C$ shows plots of $\mathrm{dt} / \mathrm{P}_{\text {applied }}$ versus $\Delta \mathrm{T} / \mathrm{P}_{\text {natural }}$ for two values of $\mathrm{P}_{\text {applied }}$ (shown in Fig. 5). Both plots show that the PSP peak phase was significantly correlated with the PY phase in the ongoing rhythm (Fig. 7B, $y=0.6 x+0.01 ; r=0.667 ; p<$ $0.05 ; n=12$ ) (Fig. $7 C, y=0.41 x+0.07 ; r=0.644 ; p<0.05 ; n=$ 12). These correlations indicate that a later peak in the $\mathrm{LP} \rightarrow \mathrm{PY}$ PSP corresponded to a later burst phase (with respect to the LP neuron) of the PY neuron in the ongoing rhythm, and that this relationship held regardless of the cycle period of the applied LP neuron waveform used to measure the PSP.

\section{Discussion}

Coordination between the firing times of different motoneurons participating in a rhythmic motor behavior is vital for producing meaningful behavior. When cycle period changes, the intervals between firing times of motoneurons must be adjusted to maintain the basic structure of the pattern. The pyloric circuit in $P$. 
interruptus is an example in which cycle period can change several-fold, yet the triphasic structure of the rhythm is relatively well conserved. In this work, we described the temporal dynamics of a mixed synapse between two members of this network, the LP and PY neurons, and showed how these complex dynamics may affect the phasing between the two neurons in response to changes in cycle period.

The synapse from the LP to the PY neuron consists of an inhibitory chemical component that shows depression and a frequency-independent electrical component. We show that when the LP neuron is activated with low frequencies, the $\mathrm{LP} \rightarrow \mathrm{PY}$ synapse has an initial inhibitory (hyperpolarizing) and a later excitatory (depolarizing) component. At higher frequencies, the effect of the LP $\rightarrow$ PY synapse is always excitatory. To our knowledge, our study is the first example to show that such a synapse can reverse its sign as an automatic response to a change in frequency of the presynaptic neuron.

In this study, we ignored the effects of spike-mediated transmission, whereas in an ongoing rhythm, the LP neuron action potentials produce both a chemical and an electrical effect on the PY neurons. In particular, because the electrical PSP caused by presynaptic action potentials may be highly filtered compared with the chemical PSP, by using low-pass-filtered realistic waveforms, we may underestimate the contribution of the chemical component. This problem may also be compounded by spaceclamp attenuation of the waveform injected at the soma, because the chemical (but not electrical) component of transmission follows a nonlinear input-output relationship. These points do not obviate the results presented here: even if the chemical component of the mixed synapse is under-represented in our protocols, its history dependence results in a dynamic balance between chemical and electrical transmission, depending on presynaptic activity.

\section{The activity phase of PY neurons in an ongoing rhythm correlates with the balance of electrical and chemical components of the $\mathrm{LP} \rightarrow \mathrm{PY}$ synapse}

There are six to eight PY neurons in the pyloric circuit, identified by their projections to the pyloric muscles and their connectivity to other pyloric neurons. Previous studies have categorized PY neurons into two groups, those that fire early or late in the pyloric cycle (Hartline and Gassie, 1979; Eisen and Marder, 1984). However, when we computed the firing phase of different PY neurons with reference to the LP neuron firing time, we did not see two well defined groups of PY neurons but a continuum of phases. Of course, we did not record more than two PY neurons in each preparation, so the variability among preparations may have obscured the phase distinction.

Interestingly, however, we found a correlation between the characteristics of the isolated $\mathrm{LP} \rightarrow \mathrm{PY}$ synapse and the phase of the PY neuron in an ongoing rhythm. When a single voltage pulse was injected into the LP neuron, the PSP in the PY neuron switched from hyperpolarizing (chemical IPSP) to depolarizing (electrical EPSP) during the pulse. Two characteristics of this mixed PSP, the zero-cross time and the ratio of the peak of the chemical IPSP and the maximum electrical EPSP, correlated well with the bursting phase of the PY neuron. Both characteristics are determined by many factors, including the relative strengths of the chemical and electrical synapses and the rates and extent of depression and recovery of the chemical synapse. However, each of these components alone did not show any significant correlation with the bursting phase of the PY neuron.

These correlations showed that those PY neurons with a PSP that switched sign at a later time or had a stronger chemical versus electrical component tended to burst at a later phase. Hence, the variability in PY phases that we observed in ongoing pyloric rhythms could be attributable to differences in the relative strength of chemical inhibition and electrical excitation. Several possibilities could explain these differences. For instance, it is possible that the chemical and electrical components affect the PY neurons at different morphological sites, or that the electrical coupling conductance or the dynamics of the chemical component vary for different PY neurons.

Because the pyloric rhythm is spontaneously active both in vivo and in vitro, there is no transient strong influence of the chemical PSP unless the rhythm is perturbed. Such perturbations do indeed arrive in the form of episodic activity of the central pattern generator controlling the cardiac sac rhythm (Thuma and Hooper, 2003). These perturbations temporarily disrupt the pyloric rhythm. After each cardiac sac episode (period, $30 \mathrm{sec}$ to several minutes), it takes the pyloric activity several cycles to reach a steady rhythmic pattern again. Because of the complexity of the cardiac sac rhythm, we have not yet studied its effect on pyloric synapses in detail. It is possible, however, that after a cardiac sac perturbation, there is a transient strong influence of the chemical inhibitory component of the LP $\rightarrow$ PY synapse.

\section{Contribution of the $\mathrm{LP} \rightarrow \mathrm{PY}$ synapse to the activity phase of the PY neurons in an ongoing pyloric rhythm}

The ideal way to quantify the synaptic influence of the LP neuron on the PY neurons is to measure the strength (amplitude) and dynamics (time-to-peak) of the LP $\rightarrow$ PY PSP in an ongoing rhythm. However, because PY neurons receive synaptic inputs from other neurons and have voltage-gated ionic conductances, it is difficult to isolate the effect of the $\mathrm{LP} \rightarrow \mathrm{PY}$ synapse in an ongoing rhythm. As a close approximation, we played back prerecorded realistic LP neuron waveforms into the voltageclamped LP neuron and measured the postsynaptic potential in the PY neuron. Because our experimental preparation is treated with TTX, all pyloric neurons are quiescent. Thus, our procedure ensures that only the LP $\rightarrow$ PY synapse and no other pyloric synapse are activated. In contrast, in the ongoing natural pyloric rhythm, other synapses are active and they affect both LP and PY neurons. These effects are expected to have some contribution on the phasing of the PY neurons.

When the LP neuron was periodically stimulated with a realistic waveform, the LP $\rightarrow$ PY synapse depolarized the PY neuron. In the ongoing rhythm, this effect is expected to advance the PY burst. Yet the chemical component of the synapse is not totally depressed and acts to delay the burst of the PY neuron and possibly decrease its amplitude. When the LP neuron was injected with realistic waveforms of different frequencies, the PY neuron PSP peaked with some delay (that was larger with larger cycle period). This effect is attributable to the frequency-dependent dynamics of the chemical component of the $\mathrm{LP} \rightarrow \mathrm{PY}$ synapse. Thus, if cycle period increases in an ongoing rhythm, it is expected that the dynamics of the chemical inhibition act to delay the PY burst.

\section{The interaction of rectifying electrical and depressing chemical components of the $\mathrm{LP} \rightarrow \mathrm{PY}$ synapse facilitates and then delays the onset of a PY burst}

At first sight, having the same presynaptic neuron exert two opposing effects on the postsynaptic cell seems puzzling. But do these two effects occur at the same time? Recall that the electrical component of the LP $\rightarrow$ PY synapse is rectifying, such that the LP 
neuron passes current to the PY neuron only during a limited part of the cycle, when it is at more positive membrane potential than the PY neuron. Both the LP and PY neurons have oscillatory membrane potentials with the PY neuron lagging in phase. Thus, at some point in the cycle, the membrane potential of the LP neuron falls below that of the PY neuron and the electrical component of the synapse disappears. We propose that, in an ongoing rhythm, the depolarizing effect of the electrical component is prominent only during the initial stages of the LP burst and is important to induce the PY burst. In the latter part of the LP burst, the chemical component of the $\mathrm{LP} \rightarrow \mathrm{PY}$ synapse becomes more significant and acts to fine-tune the burst time of the PY neuron according to cycle frequency. We are currently investigating this hypothesis with a computational model.

\section{The mixed LP $\rightarrow$ PY synapse may help maintain constant phase between the two neurons}

Previous studies have computed the activity phase of different neurons in the pyloric cycle (Hooper, 1997a,b). In these studies, it was found that across different cycle frequencies, the LP (as well as PY) neuron burst onsets did not occur at a fixed phase with respect to the pacemaker neurons. As cycle frequency was increased from 0.5 to $2.5 \mathrm{~Hz}$, both the LP and the PY neuron bursts occurred at later phases. Interestingly, however, in this frequency range, the phase difference between the LP and PY neuron bursts was nearly constant (Hooper, 1997b). A recent modeling study suggests that in a simple circuit that consists of an oscillator coupled to a follower with an inhibitory synapse, synaptic depression is used to keep an approximately constant phase between the oscillator and the follower as cycle period is changed (Nadim et al., 2003). The present work suggests that a similar mechanism could be used to maintain an approximately fixed phase between the LP and PY neurons across different cycle periods.

\section{Conclusions}

We have shown that in an oscillatory network, a mixed synapse could have significant influences on the activity of the postsynaptic neuron. First, because the chemical component is frequency dependent and the electrical component is relatively constant, the total effect depends on the frequency, and in some cases, it can reverse its sign. Moreover, the combination of the two synaptic components dynamically affects the shape of the postsynaptic potential depending on the shape of the presynaptic waveform. Second, certain parameters describing the dynamics of the synapse correlate with the relative phasing of the presynaptic and postsynaptic neurons. We propose that, at this synapse, both excitation and inhibition are required for the normal phasing of PY neurons, but at different times. The combination of depressing chemical inhibition and rectifying electrical excitation satisfies this requirement.

\section{References}

Benardo LS (1997) Recruitment of GABAergic inhibition and synchronization of inhibitory interneurons in rat neocortex. J Neurophysiol 77:3134-3144.

Eisen JS, Marder E (1984) A mechanism for production of phase shifts in a pattern generator. J Neurophysiol 51:1375-1393.
Fukuda T, Kosaka T (2000) Gap junctions linking the dendritic network of GABAergic interneurons in the hippocampus. J Neurosci 20:1519-1528.

Galarreta M, Hestrin S (1999) A network of fast-spiking cells in the neocortex connected by electrical synapses. Nature 402:72-75.

Galarreta M, Hestrin S (2001a) Spike transmission and synchrony detection in networks of GABAergic interneurons. Science 292:2295-2299.

Galarreta M, Hestrin S (2001b) Electrical synapses between GABA-releasing interneurons. Nat Rev Neurosci 2:425-433.

Gibson JR, Beierlein M, Connors BW (1999) Two networks of electrically coupled inhibitory neurons in neocortex. Nature 402:75-79.

Graubard K, Hartline DK (1987) Full-wave rectification from a mixed electrical-chemical synapse. Science 237:535-537.

Graubard K, Raper JA, Hartline DK (1980) Graded synaptic transmission between spiking neurons. Proc Natl Acad Sci USA 77:3733-3735. mcHarris-Warrick RM, Marder E, Selverston AI, Moulins M (1992) Dynamic biological networks. The stomatogastric nervous system. Cambridge, MA: MIT.

Hartline DK, Gassie Jr DV (1979) Pattern generation in the lobster (Panulirus) stomatogastric ganglion. I. Pyloric neuron kinetics and synaptic interactions. Biol Cybern 33:209-222.

Hooper SL (1997a) Phase maintenance in the pyloric pattern of the lobster (Panulirus interruptus) stomatogastric ganglion. J Comput Neurosci 4:191-205.

Hooper SL (1997b) The pyloric pattern of the lobster (Panulirus interruptus) stomatogastric ganglion comprises two phase maintaining subsets. J Comput Neurosci 4:207-219.

Johnson BR, Peck JH, Harris-Warrick RM (1993) Dopamine induces sign reversal at mixed chemical-electrical synapses. Brain Res 625:159-164.

Mann-Metzer P, Yarom Y (1999) Electrotonic coupling interacts with intrinsic properties to generate synchronized activity in cerebellar networks of inhibitory interneurons. J Neurosci 19:3298-3306.

Manor Y, Nadim F, Abbott LF, Marder E (1997) Temporal dynamics of graded synaptic transmission in the lobster stomatogastric ganglion. J Neurosci 17:5610-5621.

Marder E, Paupardin-Tritsch D (1978) The pharmacological properties of some crustacean neuronal acetylcholine, gamma-aminobutyric acid and L-glutamate responses. J Physiol (Lond) 280:213-236.

Michelson HB, Wong RK (1994) Synchronization of inhibitory neurones in the guinea-pig hippocampus in vitro. J Physiol (Lond) 477:35-45.

Miller JP, Selverston AI (1982) Mechanisms underlying pattern generation in lobster stomatogastric ganglion as determined by selective inactivation of identified neurons. IV. Network properties of pyloric system. J Neurophysiol 48:1416-1432.

Nadim F, Booth V, Bose A, Manor Y (2003) Short-term synaptic dynamics promote phase maintenance in multi-phasic rhythms. Neurocomputing 52-54:79-87.

Nusbaum MP, Beenhakker MP (2002) A small-systems approach to motor pattern generation. Nature 417:343-350.

Olsen OH, Calabrese RL (1996) Activation of intrinsic and synaptic currents in leech heart interneurons by realistic waveforms. J Neurosci 16:4958-4970.

Raper JA (1979) Nonimpulse-mediated synaptic transmission during the generation of a cyclic motor program. Science 205:304-306.

Selverston AI, Russell DF, Miller JP (1976) The stomatogastric nervous system: structure and function of a small neural network. Prog Neurobiol 7:215-290.

Simmons P (2002) Presynaptic depolarization rate controls transmission at an invertebrate synapse. Neuron 35:749.

Tamas G, Buhl EH, Lorincz A, Somogyi P (2000) Proximally targeted GABAergic synapses and gap junctions synchronize cortical interneurons. Nat Neurosci 3:366-371.

Thuma JB, Hooper SL (2003) Quantification of cardiac sac network effects on a movement-related parameter of pyloric network output in the lobster. J Neurophysiol 89:745-753. 